\title{
Association of the Pretreatment Lung Immune Prognostic Index with Survival Outcomes in Advanced Hepatocellular Carcinoma Patients Treated with PD-I Inhibitors
}

This article was published in the following Dove Press journal: Journal of Hepatocellular Carcinoma

\author{
Shixue Chen ${ }^{1,2, *}$ \\ Ziwei Huang ${ }^{2,3, *}$ \\ Wangping Jia ${ }^{1,4, *}$ \\ Haitao Tao ${ }^{2}$ \\ Sujie Zhang ${ }^{2}$ \\ Junxun $\mathrm{Ma}^{2}$ \\ Zhefeng Liu ${ }^{2}$ \\ Jinliang Wang ${ }^{2}$ \\ Lijie Wang ${ }^{2}$ \\ Pengfei Cui ${ }^{1,2}$ \\ Zhibo Zhang (iD ${ }^{1,2}$ \\ Di Huang ${ }^{2,3}$ \\ Zhaozhen $\mathrm{Wu}^{2,3}$ \\ Xuan Zheng ${ }^{1,2}$ \\ $\mathrm{Yi} \mathrm{Hu}{ }^{2}$
}

'Department of Graduate Administration, Chinese PLA General Hospital, Beijing, People's Republic of China; ${ }^{2}$ Department of Medical Oncology, Chinese PLA General Hospital, Beijing, People's Republic of China;

${ }^{3}$ School of Medicine, Nankai University, Tianjin, People's Republic of China; ${ }^{4}$ Institute of Geriatrics, Beijing Key Laboratory of Aging and Geriatrics, National Clinical Research Center for Geriatrics Diseases, Second Medical Center of Chinese PLA General Hospital, Beijing, People's Republic of China

*These authors contributed equally to this work

Correspondence: $\mathrm{Yi} \mathrm{Hu}$

Department of Medical Oncology,

Chinese PLA General Hospital, 28 Fuxing

Road, Haidian, Beijing, People's Republic

of China

Email huyi040I30I@sina.com
Purpose: At present, there are no validated biomarkers that can predict whether patients with advanced hepatocellular carcinoma (aHCC) are likely to benefit from anti-PD-1 therapy. We aimed to determine whether lung immune prognostic index (LIPI) is associated with outcomes in patients with aHCC treated with PD-1 inhibitors.

Patients and Methods: Patients undergoing initial treatment with PD-1 inhibitors for aHCC at a single center from January 1, 2015 to August 31, 2019 were included. The patients were stratified according to pretreatment LIPI based on a derived neutrophils/ (leukocytes minus neutrophils) ratio (dNLR) $\geq 3$ and a lactate dehydrogenase (LDH) level $\geq$ the upper limit of normal (ULN). Kaplan-Meier analysis and the Log rank test were used to calculate and compare survival between good LIPI and intermediate/poor LIPI scores. The prognostic values of LIPI for survival and disease control rate were evaluated using Cox proportional hazard and logistic regression models, respectively.

Results: Of the 108 study patients, 53 (49\%) had a good LIPI (dNLR $<3$ and LDH normal) and $55(51 \%)$ had intermediate/poor LIPI (dNLR $\geq 3$ or/and LDH $\geq U L N)$. With a median follow-up of 12.4 months, intermediate/poor LIPI was independently associated with shorter overall survival (OS) (hazard ratio [HR] 4.00; 95\% CI, 2.00-8.03) and progression-free survival (PFS) (HR 2.65; 95\% CI, 1.61-4.37). The median OS for good and intermediate/ poor LIPI was not reached and was 13.7 (95\% CI, 8.2-19.1) months, respectively, and the median PFS was 10.9 (95\% CI, 8.9-12.9) and 4.0 (95\% CI, 2.2-5.8) months (both $\mathrm{P}<$ $0.001)$, respectively.

Conclusion: Pretreatment LIPI combined with a dNLR $\geq 3$ and/or LDH $\geq$ ULN is associated with poor outcomes in patients with aHCC treated with PD-1 inhibitors. Further validation in large, prospective studies are warranted.

Keywords: PD-1 inhibitor, hepatocellular carcinoma, biomarker, LIPI, dNLR, LDH

\section{Introduction}

Immunotherapies targeting immune checkpoints are being rapidly developed as therapeutic approaches. Checkpoint inhibitors targeting programmed cell death protein 1 (PD-1) have been applied for various tumor types, triggering durable responses and prolonging survival. PD-1 inhibitors, such as pembrolizumab and nivolumab, have become a promising treatment option for various cancers and have been approved by the FDA for the front-line treatment of advanced hepatocellular 
carcinoma (aHCC). ${ }^{1,2}$ However, PD-1 inhibitors only benefit a small proportion of HCC patients and are associated with a wide range of immune-related adverse events (IrAEs), highlighting the urgent need to identify biomarkers that can predict patients who are likely to respond to anti-PD-1 therapy. Unlike lung cancer and melanoma, in which immunotherapies have been widely used, there remains a lack of study regarding prognosis biomarkers for patients with aHCC receiving PD-1 inhibitors.

The expression of programmed cell death ligand 1 (PDL1) on tumor cells has a certain but not absolute correlation with the efficacy of PD-1 inhibitors approved by the FDA in various tumors, including lung cancer and urothelial carcinoma. However, this relationship has not been validated in HCC. Regardless of the expression of PD-L1, the irrespective response can be observed, indicating that PD-L1 is not a sufficiently reliable biomarker for patients with HCC.,3 Another approved biomarker, tumor mutation burden (TMB), has limited clinical application owing to its heterogeneity and lack of standardized testing. Because of the extremely low frequency of mismatch repair (MMR) protein deficiencies and microsatellite instability (MSI) in patients with HCC, their utilities are also restricted. ${ }^{4}$ Furthermore, the above biomarkers must all be determined via gene testing or immunohistochemistry, which are not routine tests and may not be cost-effective. Therefore, several previous studies have focused on exploring the available biomarkers in serology that may predict outcomes in patients treated with PD-1 inhibitors.

Mezquita and colleagues initiated the use of lung immune prognostic index (LIPI), which is simply based on a derived neutrophil-to-lymphocyte ratio (dNLR) $\geq 3$ and a pretreatment lactate dehydrogenase (LDH) level $\geq$ the upper limit of normal (ULN), as a prognosis biomarker for patients with non-smallcell lung cancer (NSCLC) treated with immune checkpoint inhibitors (ICIs). ${ }^{5}$ Several studies have demonstrated the prognostic value of LIPI in renal cell carcinoma, melanoma, and NSCLC. Intermediate/poor LIPI at baseline is associated with treatment failure and increased risk of death in these studies. ${ }^{6-9}$ As such, we evaluated the prognostic value of LIPI on survival outcomes in patients with aHCC treated with PD-1 inhibitors using real-world data.

\section{Patients and Methods}

\section{Patients}

Institutional review board approval was acquired to review medical records at the Chinese People's Liberation Army General Hospital (approval number: S2018-092-01). All patient data accessed complied with relevant data protection and privacy regulations. Informed consent was waived by the Ethics Committee of Chinese People's Liberation Army General Hospital owing to the retrospective nature of this study. Following the national legislation and institutional requirements, written informed consent for participation was not required for this study. In addition, this paper does not contain any individual person's data in any form. A total of 129 consecutive patients with aHCC who received PD-1 inhibitors between January 1, 2015 and August 31, 2019 were reviewed, of whom 108 patients were included in the final analysis. Information on complete blood cell counts with differential counts and LDH levels within 14 days before the first anti-PD-1 treatment were extracted. In addition, demographic, clinical, and pathological data were collected. Tumor assessment was performed at baseline and then after every two treatment cycles, which was generally after every 6 weeks. Radiographic responses were classified according to the response evaluation criteria in solid tumors (RECIST 1.1).

LIPI scores, which comprise two factors, were calculated according to dNLR (absolute neutrophil count/[white blood cell count - absolute neutrophil count] $) \geq 3$ and LDH levels $\geq$ ULN, as reported by Mezquita et al. ${ }^{5}$ In our study, we divided the patients into two groups: those with good LIPI (dNLR $<3$ and LDH normal) and those with intermediate/poor LIPI (intermediate, $\mathrm{dNLR} \geq 3$ or $\mathrm{LDH} \geq \mathrm{ULN}$; poor, $\mathrm{dNLR} \geq 3$ and LDH $\geq$ ULN) owing to the limited number of patients with aHCC receiving immunotherapy.

\section{Statistical Analysis}

Overall survival (OS) was calculated from the first PD-1 inhibitor treatment until death or till the last follow-up (censored). Progression-free survival (PFS) was calculated from the first PD-1 inhibitor treatment until the first documented disease progression (PD), death, or the last follow-up (censored). The objective response rate (ORR) was the number of patients with best responses of complete response (CR) or partial response (PR). The disease control rate (DCR) was the number of patients with $\mathrm{CR}, \mathrm{PR}$, or stable disease.

The clinical characteristics of the patients were compared using the Fisher's exact test for discrete variables and the Wilcoxon rank-sum test for continuous variables. Survival analyses were performed using the Kaplan-Meier method and Log rank test. Multivariate Cox proportional PFS and OS hazard ratios (HRs) were fitted based on significant univariate factors. Covariates associated with disease control were assessed using univariate and multivariate logistic regression models. All $\mathrm{P}$ values were from two-sided tests and were 
considered statistically significant at $\mathrm{P}<0.05$. IBM SPSS, version 25.0, with Stata, version 15.1 was used to perform statistical analyses.

\section{Results \\ Patient Characteristics}

In total, 108 patients with unresectable or stage IV HCC who were treated with PD-1 inhibitors were included in the study after excluding 17 patients who lacked the relevant data to be assigned to the LIPI group within 14 days of initiation of anti-PD-1 therapy and 4 patients who were lost to follow-up within 1 month. Fifty-three (49.1\%), 38 (35.2\%), and $17(15.7 \%)$ patients were allocated to the good, intermediate, and poor LIPI groups, respectively. Owing to the small number of patients in the poor LIPI group, we eventually divided the patients into two groups: the good LIPI group (dNLR $<3$ and LDH normal) with 53 (49.1\%) patients and intermediate/poor LIPI group (dNLR $\geq 3$ and/or LDH $\geq$ ULN) with 55 (50.9\%) patients.

The median age at the initiation of anti-PD-1 therapy was 56 years, and $83.3 \%$ of the patients were men (Table 1). Most patients had an Eastern Cooperative Oncology Group performance status (ECOG PS) score of 0 (65.7\%), followed by scores of $1(23.2 \%)$ or $\geq 2(11.1 \%)$. The large majority of patients were infected with hepatitis B virus (76.9\%). Twenty-eight (25.9\%) and 77 (71.3\%) patients had experienced prior targeted therapy and locoregional therapy (LRT) before the initiation of anti-PD-1 therapy, respectively. Seventy-seven (71.3\%) patients received PD-1 inhibitors as the first-line treatment. Twenty-seven $(25.0 \%)$ patients were treated with PD-1 monotherapy and $81(75.0 \%)$ were treated with PD-1 inhibitors simultaneously combined with targeted therapy. The targeted drugs for combination therapy were multitargeted tyrosine kinase inhibitors, lenvatinib (76.5\%), apatinib (11.1\%), sorafenib (7.4\%), and anlotinib (4.9\%). The most frequently used PD-1 inhibitor was nivolumab $(50.0 \%)$. The baseline patient characteristics were generally well balanced between the two groups.

\section{Association of LIPI Scores with OS}

With a median follow-up of 12.4 (95\% CI, 10.8-14.0) months, the median OS for the entire study population was 16.2 (95\% CI, 11.8-20.6) months. Good LIPI was associated with significantly longer OS compared with intermediate/ poor LIPI $(\mathrm{P}<0.001)$ (Figure 1). The median OS was not reached and was 13.7 (95\% CI, 8.2-19.1) months in the good and intermediate/poor LIPI groups, respectively.
In univariate analysis, intermediate/poor LIPI, ECOG PS scores of 1 and $\geq 2$, Child-Pugh stage C, BCLC stage D, and other PD-1 inhibitor agents were significantly associated with OS. Multivariate analysis revealed that intermediate/poor LIPI was associated with a significantly increased risk of death (HR, 4.00; 95\% CI, 2.00-8.03; P < 0.0001). ECOG PS 1 and $\geq 2$ and other PD-1 inhibitor agents were also independent prognostic factors (Table 2). The complete information from univariate analysis is listed in Supplementary Table S1.

\section{Association of LIPI Scores with PFS}

The median PFS for the overall population was $7.8(95 \% \mathrm{CI}$, 5.1-10.4) months. Good LIPI was associated with a significantly longer PFS compared with intermediate/poor LIPI $(\mathrm{P}<0.001)$ (Figure 1). The median PFS was 10.9 (95\% CI, 8.9-12.9) months and 4.0 (95\% CI, 2.2-5.8) months in the good and intermediate/poor LIPI groups, respectively.

In univariate analysis, intermediate/poor LIPI, ECOG PS scores $\geq 2$, Child-Pugh stage $C$, and combination with targeted therapy were significantly associated with PFS. In multivariate analysis, intermediate/poor LIPI emerged as the strongest unfavorable prognostic factor of PFS (HR, 2.65; 95\% CI, 1.61-4.37; P < 0.001). Combined treatment with targeted therapy was another independent prognostic factor (Table 2). The complete information from univariate analysis is listed in Supplementary Table S1.

\section{Association of LIPI Scores with Response}

As summarized in Table 3, the relationship between LIPI and DCR from anti-PD-1 treatment was significant (good vs intermediate/poor LIPI, $81.1 \%$ vs $61.8 \% ; \mathrm{P}=0.029$ ). In multivariate logistic regression analysis (Table 4), intermediate/ poor LIPI and prior targeted therapy were associated with progressive disease, with ORs of 2.20 (95\% CI, 1.03-7.13; $\mathrm{P}=0.044)$ and 3.09 (95\% CI, 1.10-8.68; $\mathrm{P}=0.032)$, respectively. Inhibitor treatment combined with targeted therapy was a favorable factor for disease control, with an OR of 0.32 (95\% CI, 0.11-0.90; $\mathrm{P}=0.030$ ). The complete information from univariate analysis is listed in Supplementary Table S2. ORR did not differ between patients with good and intermediate/ poor LIPI $(26.4 \%$ vs $16.4 \%$; $\mathrm{P}=0.242)$.

\section{Association of LIPI Scores with Outcomes in PD-I Inhibitor Monotherapy or Combination Therapy: Subgroup Analysis}

It is known that the efficacy of PD-1 inhibitor monotherapy is very different from that of PD-1 inhibitor-based combination 
Table I Baseline Patient Demographic and Clinical Characteristics

\begin{tabular}{|c|c|c|c|c|}
\hline \multirow[t]{2}{*}{ Characteristics } & \multicolumn{3}{|l|}{ No. of Patients (\%) } & \multirow[t]{2}{*}{$P$ value } \\
\hline & Overall $(n=108)$ & Good LIPI $(n=53)$ & Intermediate/Poor LIPI $(n=55)$ & \\
\hline Median age (range), years & $56(29-88)$ & $57(29-88)$ & $53(33-82)$ & 0.355 \\
\hline Sex & & & & 0.309 \\
\hline Male & $90(83.3)$ & $42(79.3)$ & $48(87.3)$ & \\
\hline Female & $18(16.7)$ & II (20.8) & $7(12.7)$ & \\
\hline ECOG performance status & & & & 0.376 \\
\hline 0 & 7I (65.7) & $38(71.7)$ & $33(60.0)$ & \\
\hline I & $25(23.2)$ & II (20.8) & $14(25.5)$ & \\
\hline$\geq 2$ & $12(11.1)$ & $4(7.6)$ & $8(14.6)$ & \\
\hline Child-Pugh stage & & & & 0.297 \\
\hline A & 85 (78.7) & $45(84.9)$ & $40(72.7)$ & \\
\hline B & $19(17.6)$ & $7(13.2)$ & $12(21.8)$ & \\
\hline C & $4(3.7)$ & I (I.9) & $3(5.5)$ & \\
\hline $\mathrm{BCLC}$ stage & & & & 0.253 \\
\hline B & $16(14.8)$ & $10(18.9)$ & $6(10.9)$ & \\
\hline C & $87(80.6)$ & $42(79.3)$ & $45(81.8)$ & \\
\hline $\mathrm{D}$ & $5(4.6)$ & I (I.9) & $4(7.3)$ & \\
\hline Viral status ${ }^{\mathrm{a}}$ & & & & 0.108 \\
\hline Uninfected & $21(19.4)$ & $9(17.0)$ & $12(21.8)$ & \\
\hline HBV & $83(76.9)$ & $44(83.0)$ & $39(70.9)$ & \\
\hline $\mathrm{HCV}$ & $4(3.7)$ & $0(0.0)$ & $4(7.3)$ & \\
\hline Extrahepatic disease & 77 (7I.3) & $39(73.6)$ & $38(69.1)$ & 0.673 \\
\hline Macrovascular invasion & $52(48.2)$ & $21(39.6)$ & $31(56.4)$ & 0.088 \\
\hline Prior target therapy & $28(26.0)$ & $13(24.5)$ & $15(27.3)$ & 0.828 \\
\hline Prior Locoregional therapy & $77(7 \mid .3)$ & $39(73.6)$ & $38(69.1)$ & 0.673 \\
\hline Baseline AFP, ng/mL & & & & 0.840 \\
\hline$<200$ & $37(34.3)$ & $19(35.9)$ & $18(32.7)$ & \\
\hline$\geq 200$ & 71 (65.7) & $34(64.2)$ & $37(67.3)$ & \\
\hline Immunotherapy as systemic & & & & 0.933 \\
\hline I & $77(7 \mid .3)$ & $37(50.9)$ & $40(72.7)$ & \\
\hline 2 & $27(25.0)$ & $14(26.4)$ & $13(23.6)$ & \\
\hline 3 & $4(3.7)$ & $2(3.8)$ & $2(3.6)$ & \\
\hline Combined with target therapy & & & & 0.659 \\
\hline No & $27(25.0)$ & $12(22.6)$ & I5 (27.3) & \\
\hline Yes & $81(75.0)$ & $4 \mathrm{I}(77.4)$ & $40(72.7)$ & \\
\hline PD-I inhibition agent & & & & 0.653 \\
\hline Nivolumab & $54(50.0)$ & $29(54.7)$ & $25(45.5)$ & \\
\hline Pembrolizumab & $26(24.1)$ & $12(22.6)$ & $14(25.5)$ & \\
\hline Others & $28(26.0)$ & $12(22.6)$ & $16(29.1)$ & \\
\hline
\end{tabular}

Notes: ${ }^{a} \mathrm{HBV}$ infection defined as hepatitis B surface antigen positive and/or detectable HBV DNA; HCV infection defined as anti-hepatitis C antibody positive and detectable HCV RNA.

Abbreviations: ECOG, Eastern Cooperative Oncology Group; BCLC, Barcelona Clinic Liver Cancer; HBV, hepatitis B virus; HCV, hepatitis C virus; AFP, a-fetoprotein.

therapy. In our study, combined with targeted therapy, PD-1 inhibitor was also an independent prognostic factor of PFS (HR, $0.55 ; 95 \%$ CI, 0.32-0.94; $\mathrm{P}=0.028$ ) (Table 2). Therefore, we conducted subgroup analysis to examine the prognostic value of pretreatment LIPI both in PD-1 inhibitor monotherapy and PD-1 inhibitor combined with targeted therapy (Figure 2). 
A

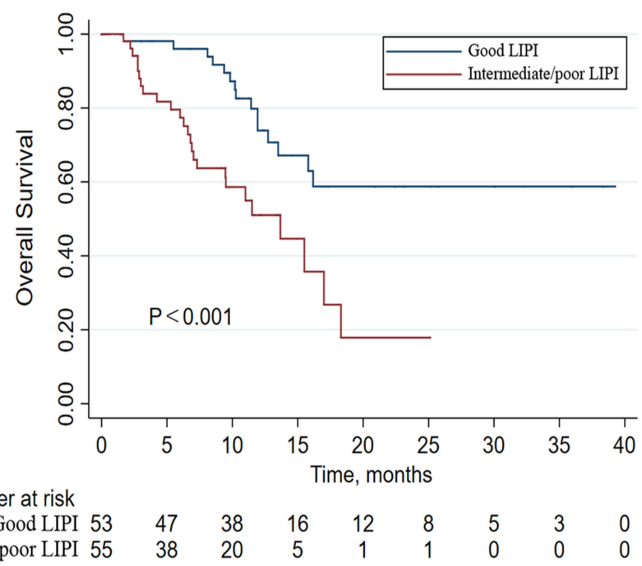

B

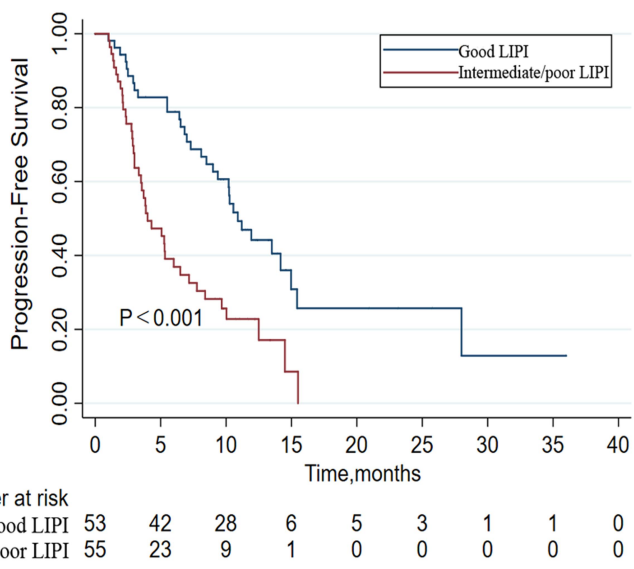

Figure I Overall survival (OS) and progression-free survival (PFS) according to lung immune prognostic index (LIPI) groups of patients with advanced hepatocellular carcinoma treated with PD-I inhibitors.

For the 27 patients treated with PD-1 inhibitor monotherapy, the median OS and PFS were 11.9 (95\% CI, 10.6-13.3) and 3.8 (95\% CI, 0.5-7.2) months, respectively. The median OS of the 12 patients in the good and 15 patients in the intermediate/poor LIPI groups was 15.8 (95\% CI, 4.5-27.1) and 7.0 (95\% CI, 5.4-8.7) months (P = 0.042), respectively, and the median PFS was 7.3 (95\% CI, 0-19.7) and 3.3 (95\% CI, 2.2-4.4) months $(\mathrm{P}=0.036)$, respectively (Figure $2 \mathrm{~A}$ and $\mathrm{B}$ ).

For the 81 patients treated with PD-1 inhibitor combined with targeted therapy, the median OS and PFS were 18.3 (95\% CI, not reached) and 10.0 (95\% CI, 8.0-12.1) months, respectively. The median OS of the 41 patients in the good and 40 patients in the intermediate/poor LIPI groups were not reached and 15.5 (95\% CI, 8.6-22.4) months $(\mathrm{P}=0.017)$, respectively, and the median PFS was 10.9 (95\% CI, 6.4-15.4) and 4.3 (95\% CI, 0.56-8.0) months $(\mathrm{P}=0.001)$, respectively (Figure $2 \mathrm{C}$ and $\mathrm{D})$.

\section{Safety}

Table 5 shows the treatment-related adverse events (TRAEs). TRAEs occurred in 92 (85.2\%) of 108 patients receiving PD-1 inhibitors, with $42(79.2 \%)$ of 53 patients in the good LIPI group and $50(90.9 \%)$ of 55 patients in the intermediate/poor LIPI group. Most TRAEs were grades 1 and 2, with a predominance of thrombocytopenia, aspartate aminotransferase (AST) increase, and blood bilirubin increase $(24.1 \%, 22.2 \%$, and $21.4 \%$ of all patients, respectively). The most frequently reported grades 3 and 4 TRAEs were thrombocytopenia in $10(9.3 \%)$ patients, AST increase in $3(2.8 \%)$, and fatigue in $3(2.8 \%)$. IrAEs occurred in $19(17.6 \%)$ of all patients, with $9(17.0 \%)$ in the good LIPI group and $10(18.2 \%)$ in the intermediate/ poor LIPI group. The most common AEs were hypothyroidism and hepatitis. AEs leading to treatment discontinuation occurred in two $(3.6 \%)$ patients of the intermediate/poor LIPI group, one of which was due to severe immune-related pneumonia and the other was due to refractory thrombocytopenia. No deaths related to antiPD-1 therapy were observed.

\section{Discussion}

PD-1 inhibition has emerged as a promising treatment approach with curable potential and durable survival; however, a large number of patients with aHCC receiving PD-1 inhibitor treatment do not experience survival benefits due to substantial heterogeneity. Biomarkers, including PD-1, TMB, and MMR/MSI, have limited predictive accuracy because of the unavailability of tumor tissue and molecular or microscopic analyses. ${ }^{10,11}$ Therefore, it is critical to explore biomarkers that can predict treatment outcomes.

A simple serum biomarker, LIPI, which is based on dNLR and LDH, was developed by Mezquita and colleagues and is considered to be associated with ICI outcomes in patients with NSCLC., 5 The prognostic relationship between higher LIPI scores and poorer outcomes has also been demonstrated in other solid tumors, including renal cell carcinoma and melanoma. ${ }^{6,9}$ Furthermore, LIPI is an ideal biomarker because it is easily available from serum and is noninvasive as well as cost-effective.

To our knowledge, this study is the first to suggest that pretreatment LIPI can be a prognostic biomarker of survival in patients with aHCC treated with PD-1 inhibitors. Our analysis demonstrated that patients treated with PD-1 
Table 2 Univariate and Multivariate Analyses of Factors Associated with Overall Survival and Progression-Free Survival

\begin{tabular}{|c|c|c|c|c|}
\hline \multirow{2}{*}{$\begin{array}{l}\text { OS } \\
\text { Characteristics }\end{array}$} & \multicolumn{2}{|c|}{ Univariate Analysis } & \multicolumn{2}{|c|}{ Multivariate Analysis } \\
\hline & HR (95\% Cl) & $\mathbf{P}$ & HR (95\% Cl) & $\mathbf{P}$ \\
\hline \multicolumn{5}{|c|}{ ECOG performance status } \\
\hline 0 & I [Ref.] & & I [Ref.] & \\
\hline I & $3.10(1.52-6.35)$ & $0.002 *$ & $4.16(1.87-9.23)$ & $<0.001 *$ \\
\hline$\geq 2$ & $5.72(2.53-12.94)$ & $<0.000 I^{*}$ & $7.17(2.2 I-23.25)$ & $0.001 *$ \\
\hline \multicolumn{5}{|l|}{ Child-Pugh stage } \\
\hline A & I [Ref.] & & I [Ref.] & \\
\hline B & $0.93(0.38-2.23)$ & 0.863 & $0.50(0.19-1.35)$ & 0.172 \\
\hline $\mathrm{C}$ & $7.41(2.15-25.60)$ & $0.002 *$ & $2.49(0.63-9.84)$ & 0.192 \\
\hline \multicolumn{5}{|l|}{$\mathrm{BCLC}$ stage } \\
\hline B & I [Ref.] & & I [Ref.] & \\
\hline C & $0.86(0.38-1.97)$ & 0.729 & $0.72(0.3 \mathrm{I}-\mathrm{I} .70)$ & 0.456 \\
\hline $\mathrm{D}$ & $4.63(1.34-16.04)$ & $0.016^{*}$ & $0.72(0.13-3.94)$ & 0.703 \\
\hline \multicolumn{5}{|l|}{ PD-I inhibition agent } \\
\hline Nivolumab & I [Ref.] & & I [Ref.] & \\
\hline Pembrolizumab & $1.03(0.50-2.10)$ & 0.945 & $0.57(0.24-1.36)$ & 0.2 \\
\hline Others & $0.29(0.09-0.96)$ & $0.042 *$ & $0.16(0.04-0.61)$ & $0.007^{*}$ \\
\hline \multicolumn{5}{|l|}{ LIPI } \\
\hline Good & I [Ref.] & & I [Ref.] & \\
\hline Intermediate/poor & $2.86(1.49-5.49)$ & $0.002 *$ & $4.00(2.00-8.03)$ & $<0.000 I^{*}$ \\
\hline PFS & \multicolumn{2}{|c|}{ Univariate Analysis } & \multicolumn{2}{|c|}{ Multivariate Analysis } \\
\hline Characteristics & HR(95\% Cl) & $\mathbf{P}$ & HR(95\% Cl) & $\mathbf{P}$ \\
\hline \multicolumn{5}{|c|}{ ECOG performance status } \\
\hline 0 & I [Ref.] & & I [Ref.] & \\
\hline I & $\mathrm{I} .4 \mathrm{I}(0.8 \mathrm{I}-2.45)$ & 0.223 & $1.28(0.70-2.34)$ & 0.43 \\
\hline$\geq 2$ & $2.27(1.14-4.53)$ & $0.020^{*}$ & I.86 (0.85-4.07) & 0.12 \\
\hline \multicolumn{5}{|l|}{ Child-Pugh stage } \\
\hline A & I [Ref.] & & I [Ref.] & \\
\hline B & $1.03(0.55-1.93)$ & 0.918 & $0.98(0.50-1.92)$ & 0.941 \\
\hline $\mathrm{C}$ & $3.2(1.15-8.93)$ & $0.027^{*}$ & $1.81(0.58-5.60)$ & 0.305 \\
\hline \multicolumn{5}{|c|}{ Combined with target therapy } \\
\hline No & I [Ref.] & & I [Ref.] & \\
\hline Yes & $0.5 \mathrm{I}(0.3 \mathrm{I}-0.85)$ & $0.009 *$ & $0.55(0.32-0.94)$ & $0.028 *$ \\
\hline \multicolumn{5}{|l|}{ LIPI } \\
\hline Good & I [Ref.] & & I [Ref.] & \\
\hline Intermediate/poor & $2.5 \mathrm{I}(1.55-4.06)$ & $<0.00 I^{*}$ & $2.65(\mathrm{I} .6 \mathrm{I}-4.37)$ & $<0.00 I^{*}$ \\
\hline
\end{tabular}

Note: $* \mathrm{P}<0.05$, statistically significant.

inhibitors with good LIPI have significantly improved OS, PFS, and DCR compared with those with intermediate/ poor LIPI. The prognostic value of LIPI in aHCC was consistent with that of previous studies in solid tumors. ${ }^{5-9}$

As we all know, systemic inflammation is closely associated with the prognosis of immunotherapy. The serologic data of potential inflammatory parameters are associated with poor prognosis in various tumors, including elevated neutrophils, NLR, dNLR, platelets, platelet-lymphocyte ratio (PLR), and LDH level. ${ }^{12-15}$ dNLR includes monocytes and other granulocyte subpopulations in addition to lymphocytes and can thus provide more immune information than NLR. dNLR has been investigated as an unfavorable prognostic factor in patients with solid tumors, such 
Table 3 Relationship Between LIPI Groups and Response to Anti-PD-I Treatment

\begin{tabular}{|c|c|c|c|c|}
\hline \multirow[t]{2}{*}{ Best Overall Response } & \multicolumn{3}{|c|}{ No. of Patients (\%) } & \multirow[t]{2}{*}{$P$ value } \\
\hline & Overall $n=108$ & Good LIPI n = 53 & Intermediate/Poor LIPI n = 55 & \\
\hline CR & $0(0)$ & $0(0)$ & $0(0)$ & \\
\hline PR & $23(21.3)$ & $14(26.4)$ & $9(16.4)$ & \\
\hline SD & $54(50.0)$ & $29(54.7)$ & $25(45.5)$ & \\
\hline PD & $29(26.9)$ & $9(17.0)$ & $20(36.4)$ & \\
\hline Not assessable ${ }^{a}$ & $2(1.9)$ & $I(1.9)$ & $\mathrm{I}(\mathrm{I} .8)$ & \\
\hline Objective response & $23(21.3)$ & $14(26.4)$ & $9(16.4)$ & 0.242 \\
\hline Disease control rate & $77(7 \mid .3)$ & $43(8 I .1)$ & $34(61.8)$ & $0.029 *$ \\
\hline
\end{tabular}

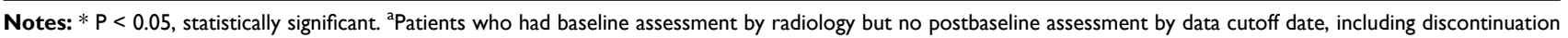
or death before first postbaseline scan.

Table 4 Logistic Regression of Disease Control Rate

\begin{tabular}{|c|c|c|c|c|}
\hline \multirow[t]{2}{*}{ Characteristics } & \multicolumn{2}{|c|}{ Univariate Analysis } & \multicolumn{2}{|c|}{ Multivariate Analysis } \\
\hline & OR (95\% CI) & $\mathbf{P}$ & OR (95\% Cl) & $\mathbf{P}$ \\
\hline \multicolumn{5}{|c|}{ ECOG performance status } \\
\hline 0 & I [Ref.] & & I [Ref.] & \\
\hline I & $1.42(0.50-4.01)$ & 0.513 & $1.16(0.37-3.66)$ & 0.796 \\
\hline$\geq 2$ & $4.13(1.11-15.30)$ & $0.034^{*}$ & $2.84(0.64-12.54)$ & 0.168 \\
\hline \multicolumn{5}{|l|}{ Prior target therapy } \\
\hline No & I [Ref.] & & I [Ref.] & \\
\hline Yes & $2.92(1.15-7.39)$ & $0.024 *$ & $3.09(1.10-8.68)$ & $0.032 *$ \\
\hline \multicolumn{5}{|c|}{ Combined with target therapy } \\
\hline No & I [Ref.] & & I [Ref.] & \\
\hline Yes & $0.27(0.11-0.70)$ & $0.006 *$ & $0.32(0.11-0.90)$ & $0.030 *$ \\
\hline \multicolumn{5}{|l|}{ LIPI } \\
\hline Good & I [Ref.] & & I [Ref.] & \\
\hline Intermediate/poor & $2.81(1.14-6.96)$ & $0.025^{*}$ & $2.70(1.03-7.13)$ & $0.044 *$ \\
\hline
\end{tabular}

Note: $* \mathrm{P}<0.05$, statistically significant.

as NSCLC, melanoma, bladder, and renal cancer. ${ }^{13,16-18}$ Furthermore, LDH, with a potential function of evaluating tumor burden, has been widely studied as an inflammatory indicator and is considered an unfavorable prognostic factor in patients with cancer. ${ }^{15,19,20}$

Recently, Dharmapuri et al demonstrated that a higher pretreatment NLR $(\mathrm{P}=0.004)$, higher PLR $(\mathrm{P}=0.05)$, and combination of higher NLR and PLR $(\mathrm{P}<0.001)$ were associated with an increased risk of death in 103 patients with HCC treated with PD-1 inhibitors. However, NLR and PLR failed to be associated with responses and PFS. ${ }^{21}$ Our study suggested that higher LIPI scores (intermediate/ poor) were an unfavorable prognostic biomarker of OS, PFS, and disease control in 108 patients with aHCC.

Notably, our observation contained a group of patients who would be excluded in most clinical trials, including patients with an ECOG PS score of $\geq 2$, Child-Pugh class C, or BCLC stage D. Our multivariate analysis revealed that an ECOG PS score of 1 (HR, 4.16; 95\% CI, 1.87-9.23; $\mathrm{P}<0.001)$ and $\geq 2$ (HR, 7.17; 95\% CI, 2.21-23.25; $\mathrm{P}<0.001$ ), in addition to intermediate/poor LIPI scores (HR, 4.00; 2.00-8.03; $\mathrm{P}<0.0001$ ), were significant and independent poor prognosis factors of OS. Conversely, Child-Pugh class C (HR, 2.49; 0.63-9.84; $\mathrm{P}=0.192)$ and BCLC stage D (HR, 0.72; 0.13-3.94; $\mathrm{P}<0.703$ ) had no significant relationship with OS. We know that patients encountered during real-world practice are far more complicated and diversified than those included in clinical trials. Therefore, our research from real-world data can provide an alternative reference for patients with similar conditions in clinical practice for whom relevant data in clinical trials are lacking. 
A

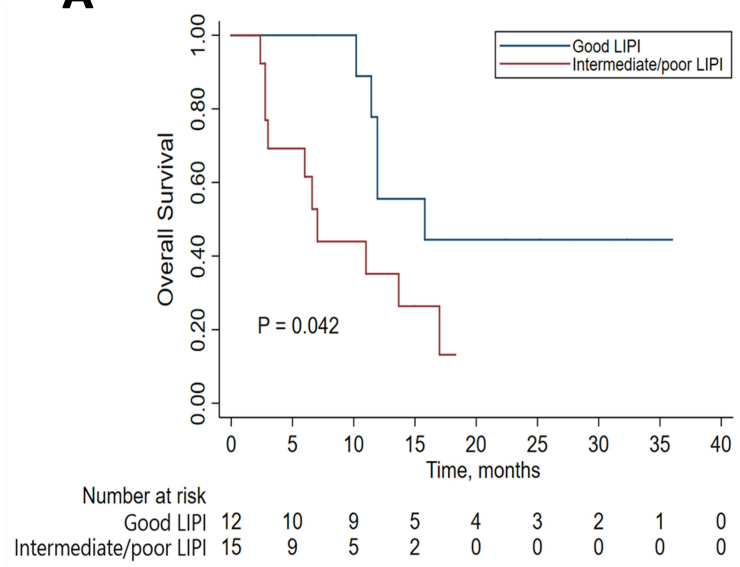

\section{C}

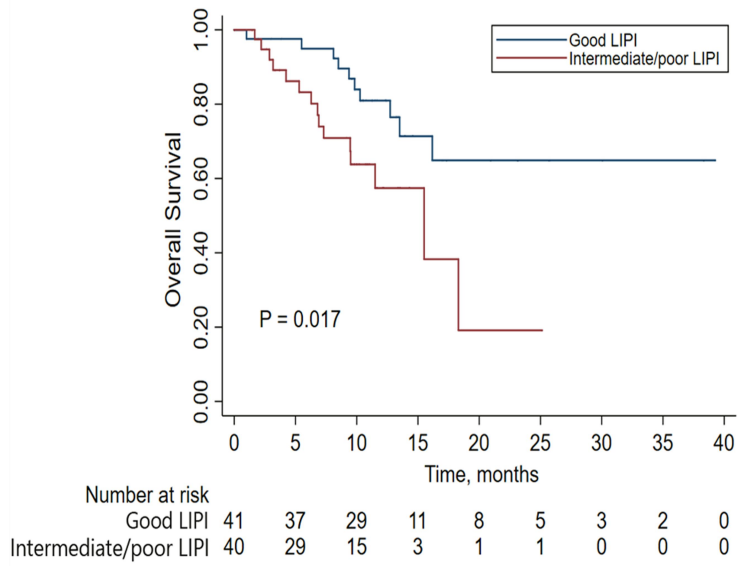

\section{B}
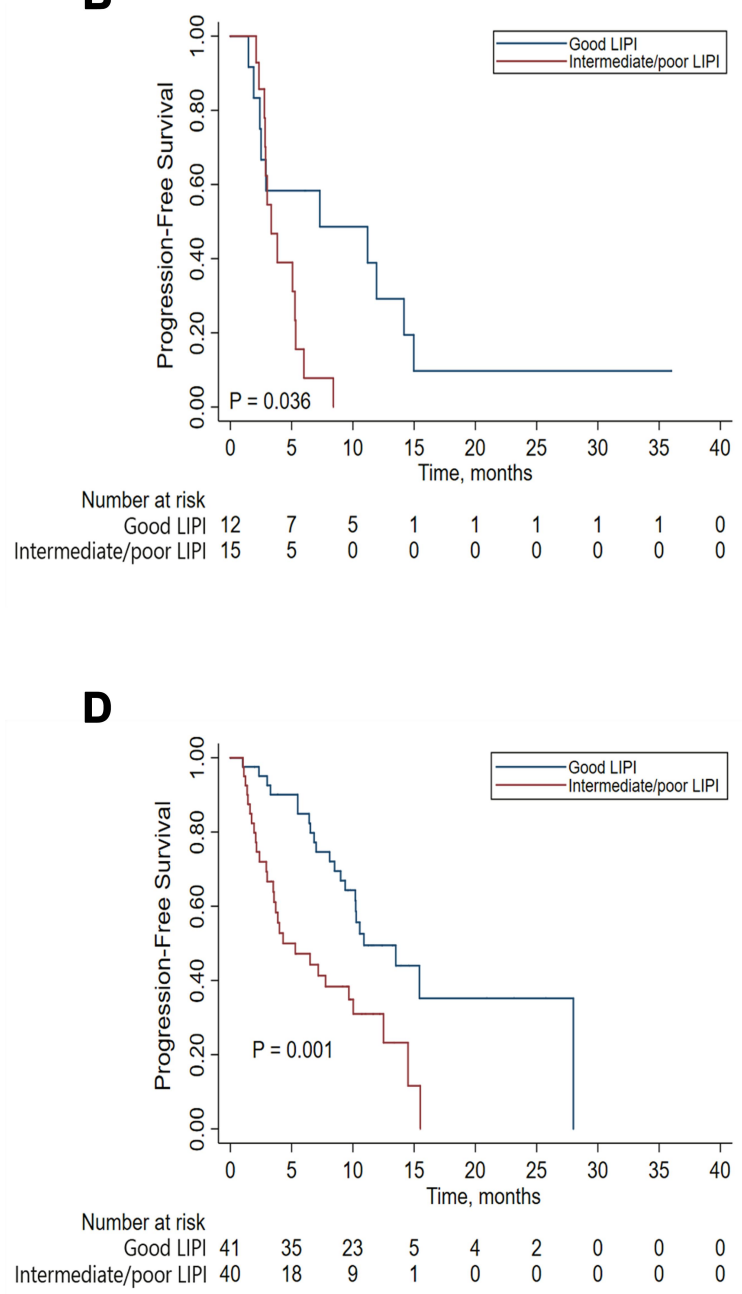

Figure 2 Overall survival (OS) and progression-free survival (PFS) according to lung immune prognostic index (LIPI) groups of patients with advanced hepatocellular carcinoma treated with PD-I inhibitors. (A) OS and (B) PFS of the PD-I inhibitor monotherapy cohort and (C) OS and (D) PFS of the PD-I inhibitor combined with targeted therapy cohort.

In this study, the PD-1 inhibitor agents (others) were four PD-1 inhibitors produced by Chinese pharmaceutical companies, ie, sintilimab (17 patients), camrelizumab (8 patients), toripalimab (2 patients), and tislelizumab (1 patient). There are two possible reasons why PD-1 inhibitor agents (others) have better OS than nivolumab or pembrolizumab. First, 89\% (25/28) patients were treated with PD-1 inhibitors simultaneously combined with targeted therapy in the others group, which was higher than that in the nivolumab (37/54 patients, 69\%) or pembrolizumab group (19/26 patients, $73 \%$ ). It is known that the efficacy of PD-1/PD-L1 inhibitor-based combination therapy (such as IMbrave $150^{22}$ or Keynote- $524^{23}$ ) is promising and is better than that of PD1 inhibitor monotherapy (such as Checkmate- $040^{1}$ and Keynote- $224^{24}$ ). Second, $86 \%$ (24/28) patients received PD-1 as first-line treatment, which was higher than those who received nivolumab (34/54 patients, $63 \%)$ or pembrolizumab (19/26 patients, $73 \%)$.

In addition, $71.3 \%$ of patients had prior LRT before the initiation of anti-PD-1 therapy in this study. LRT has long been a vital part of HCC treatment and are available for patients in whom resection or transplant is not a viable option. Ablation, chemoembolization, and radioembolization are examples of commonly used treatment techniques for HCC. It has been noted for many years that LRT may have additional systemic effects other than simple tumor elimination. In addition, LRT could induce inflammation, thermocoagulation, and other DNA-disturbing activities that create conditions that can stimulate the release of tumor-associated antigens and neoantigens into the bloodstream, which would enhance the sensitivity to checkpoint inhibitors and induce immune responses. ${ }^{25,26}$ With the 
Table 5 Treatment-Related Adverse Events

\begin{tabular}{|c|c|c|c|c|c|c|}
\hline \multirow[t]{3}{*}{ AE } & \multicolumn{6}{|l|}{ No. (\%) } \\
\hline & \multicolumn{2}{|c|}{ All Patients $(n=108)$} & \multicolumn{2}{|c|}{ Good LIPI (n=53) } & \multicolumn{2}{|c|}{ Intermediate/Poor LIPI $(n=55)$} \\
\hline & Any Grade & Grade 3/4 & Any Grade & Grade $3 / 4$ & Any Grade & Grade 3/4 \\
\hline Any & $92(85.2)$ & $30(27.8)$ & $42(79.2)$ & $13(24.5)$ & $50(90.9)$ & $17(30.9)$ \\
\hline Leading to discontinuation of treatment & $2(1.9)$ & $2(1.9)$ & $0(0)$ & $0(0)$ & $2(3.6)$ & $2(3.6)$ \\
\hline Leading to death attributed to treatment & $0(0)$ & $0(0)$ & $0(0)$ & $0(0)$ & $0(0)$ & $0(0)$ \\
\hline \multicolumn{7}{|l|}{ Occurring in $\geq 5 \%$ of all patients, any grade ${ }^{a}$} \\
\hline Thrombocytopenia & $26(24.1)$ & $10(9.3)$ & $12(22.6)$ & $5(9.4)$ & $14(25.5)$ & $5(9.1)$ \\
\hline AST increase & $24(22.2)$ & $3(2.8)$ & II (20.8) & $0(0)$ & $13(23.6)$ & $3(5.5)$ \\
\hline Blood bilirubin increase & $23(21.4)$ & $2(1.9)$ & $8(15.1)$ & $0(0)$ & $15(27.3)$ & $2(3.6)$ \\
\hline Hypoalbuminaemia & $21(19.4)$ & $0(0)$ & $10(18.9)$ & $0(0)$ & II (20.0) & $0(0)$ \\
\hline ALT increase & $20(18.5)$ & $\mathrm{I}(0.9)$ & $8(15.1)$ & $0(0)$ & $12(21.8)$ & $\mathrm{I}(\mathrm{I} .8)$ \\
\hline Pruritus & $19(17.6)$ & I (0.9) & II (20.8) & I (I.9) & $8(14.5)$ & $0(0)$ \\
\hline Diarrhea & $17(15.7)$ & $0(0)$ & $7(13.2)$ & $0(0)$ & $10(18.2)$ & $0(0)$ \\
\hline Anaemia & $16(14.8)$ & $2(1.9)$ & $6(11.3)$ & I (I.9) & $10(18.2)$ & $\mathrm{I}(1.8)$ \\
\hline Fatigue & $13(12.0)$ & $3(2.8)$ & $5(9.4)$ & I (I.9) & $8(14.5)$ & $2(3.6)$ \\
\hline Decreased appetite & $12(11.1)$ & $\mathrm{I}(0.9)$ & $2(3.8)$ & $0(0)$ & $10(18.2)$ & $\mathrm{I}(\mathrm{I} .8)$ \\
\hline Rash & $10(9.3)$ & $0(0)$ & $4(7.5)$ & I (I.9) & $6(10.9)$ & $0(0)$ \\
\hline Neutropenia & $8(7.4)$ & $0(0)$ & $4(7.5)$ & $0(0)$ & $4(7.3)$ & $0(0)$ \\
\hline \multicolumn{7}{|l|}{ Events of interest ${ }^{\mathrm{b}}$} \\
\hline Any & $19(17.6)$ & $4(3.7)$ & $9(17.0)$ & $2(3.8)$ & $10(18.2)$ & $2(3.6)$ \\
\hline Hypothyroidism & $8(7.4)$ & $0(0)$ & $3(5.7)$ & $0(0)$ & $5(9.1)$ & $0(0)$ \\
\hline Hepatitis & $6(5.6)$ & $2(1.9)$ & $2(3.8)$ & I (1.9) & $4(7.3)$ & I (I.8) \\
\hline Thyroiditis & $2(1.9)$ & $0(0)$ & $2(3.8)$ & $0(0)$ & $0(0)$ & $0(0)$ \\
\hline Pneumonitis & $2(1.9)$ & $\mathrm{I}(0.9)$ & I (I.9) & $0(0)$ & I (I.8) & $\mathrm{I}(\mathrm{I} .8)$ \\
\hline Severe skin reaction & I (0.9) & I (0.9) & I (I.9) & I (I.9) & $0(0)$ & $0(0)$ \\
\hline
\end{tabular}

Notes: ${ }^{a}$ Events listed in descending order of frequency in all patients group. ${ }^{\mathrm{b}}$ Events of interest are those with immune-related cause considered regardless of attribution to study treatment; listed in all patients group.

Abbreviations: AE, adverse event; AST, aspartate aminotransferase; ALT, alanine aminotransferase.

advent of immunotherapy for HCC, there is an increasing interest in exploring the potential synergistic effects of anti-PD-1/antiPD-L1 when used in combination with LRT.

This study has several limitations. First, our exploratory evaluation was retrospective and post-hoc in nature, resulting in inevitable biases because of patients being lost to followup and some patients being unable to be included in the analysis due to missing pretreatment laboratory data. Second, analysis was performed using data from a single institution and enrolled a small number of patients. Therefore, we stratified all patients into two groups (good and intermediate/poor LIPI) instead of three groups (good, intermediate, and poor LIPI) to perform analyses. In addition, $76.9 \%$ of patients in this study had chronic hepatitis B virus infection. This patient cohort is unique for China and is not reproducible to other parts of the world. Therefore, the results should be further validated in other regions where the aHCC cohort dominantly comprises HCC patients with hepatitis C, metabolic syndrome, or alcohol in their background. Another limitation was the lack of a control cohort not receiving PD-1 inhibitor treatment, which means that we were unable to determine whether LIPI is a prognostic biomarker only of PD-1 inhibition or a general prognostic biomarker for other treatments. Furthermore, the optimal threshold of dNLR and LDH in patients with aHCC and a better prediction model combing LIPI with other predictive indexes, such as PD-1 expression and TMB, warrant further identification in a large, prospective cohort.

\section{Conclusion}

This study is the first to suggest that pretreatment LIPI is an independent unfavorable prognostic biomarker of outcomes in patients with aHCC treated with PD-1 inhibitors. Further large, prospective studies are warranted to better understand the prognostic value of LIPI. 


\section{Funding}

This work was supported by grants from the Natural Science Foundation of Beijing (No.18BJZ28). The authors would like to thank all study patients and their family members.

\section{Disclosure}

Shixue Chen, Ziwei Huang, and Wangping Jia are co-first authors for this study. The authors report no conflicts of interest in this work.

\section{References}

1. El-Khoueiry AB, Sangro B, Yau T, et al. Nivolumab in patients with advanced hepatocellular carcinoma (CheckMate 040): an open-label, non-comparative, Phase $1 / 2$ dose escalation and expansion trial. Lancet. 2017;389(10088):2492-2502. doi:10.1016/s0140-6736(17) 31046-2

2. Finn RS, Ryoo BY, Merle P, et al. Pembrolizumab as second-line therapy in patients with advanced hepatocellular carcinoma in KEYNOTE-240: a randomized, double-blind, Phase III trial. J Clin Oncol. 2020;38(3):193-202. doi:10.1200/jco.19.01307

3. Kudo M. Pembrolizumab for the treatment of hepatocellular carcinoma. Liver Cancer. 2019;8(3):143-154. doi:10.1159/ 000500143

4. Goumard C, Desbois-Mouthon C, Wendum D, et al. Low levels of microsatellite instability at simple repeated sequences commonly occur in human hepatocellular carcinoma. Cancer Genomics Proteomics. 2017;14:329-339. doi:10.21873/cgp.20043

5. Mezquita L, Auclin E, Ferrara R, et al. Association of the lung immune prognostic index with immune checkpoint inhibitor outcomes in patients with advanced non-small cell lung cancer. JAMA Oncol. 2018;4(3):351-357. doi:10.1001/jamaoncol.2017.4771

6. Meyers DE, Stukalin I, Vallerand IA, et al. The lung immune prognostic index discriminates survival outcomes in patients with solid tumors treated with immune checkpoint inhibitors. Cancers. 2019;11 (11):11. doi:10.3390/cancers11111713

7. Sorich MJ, Rowland A, Karapetis CS, Hopkins AM. Evaluation of the lung immune prognostic index for prediction of survival and response in patients treated with atezolizumab for NSCLC: pooled analysis of clinical trials. $J$ Thorac Oncol. 2019;14(8):1440-1446. doi:10.1016/j.jtho.2019.04.006

8. Kazandjian D, Gong Y, Keegan P, Pazdur R, Blumenthal GM. Prognostic value of the lung immune prognostic index for patients treated for metastatic non-small cell lung cancer. JAMA Oncol. 2019;5(10):1481. doi:10.1001/jamaoncol.2019.1747

9. Benitez JC, Recondo G, Rassy E, Mezquita L. The LIPI score and inflammatory biomarkers for selection of patients with solid tumors treated with checkpoint inhibitors. $Q \mathrm{~J}$ Nucl Med Mol Imaging. 2020;64(2):162-174. doi:10.23736/s1824-4785.20.03250-1

10. Ready N, Hellmann MD, Awad MM, et al. First-line nivolumab plus ipilimumab in advanced non-small-cell lung cancer (CheckMate 568): outcomes by programmed death ligand 1 and tumor mutational burden as biomarkers. J Clin Oncol. 2019;37(12):992-1000. doi:10.1200/JCO.18.01042
11. Khemlina G, Ikeda S, Kurzrock R. The biology of hepatocellular carcinoma: implications for genomic and immune therapies. Mol Cancer. 2017;16(1):149. doi:10.1186/s12943-017-0712-x

12. Bagley SJ, Kothari S, Aggarwal C, et al. Pretreatment neutrophil-tolymphocyte ratio as a marker of outcomes in nivolumab-treated patients with advanced non-small-cell lung cancer. Lung Cancer. 2017;106:1-7. doi:10.1016/j.lungcan.2017.01.013

13. Russo A, Franchina T, Ricciardi GRR, et al. Baseline neutrophilia, derived neutrophil-to-lymphocyte ratio (dNLR), platelet-tolymphocyte ratio (PLR), and outcome in non small cell lung cancer (NSCLC) treated with nivolumab or docetaxel. J Cell Physiol. 2018;233(10):6337-6343. doi:10.1002/jcp.26609

14. Kargl J, Busch SE, Yang GH, et al. Neutrophils dominate the immune cell composition in non-small cell lung cancer. Nat Commun. 2017;8 (1):14381. doi:10.1038/ncomms 14381

15. Diem S, Kasenda B, Spain L, et al. Serum lactate dehydrogenase as an early marker for outcome in patients treated with anti-PD-1 therapy in metastatic melanoma. $\mathrm{Br} J$ Cancer. 2016;114 (3):256-261. doi:10.1038/bjc.2015.467

16. Ferrucci PF, Ascierto PA, Pigozzo J, et al. Baseline neutrophils and derived neutrophil-to-lymphocyte ratio: prognostic relevance in metastatic melanoma patients receiving ipilimumab. Ann Oncol. 2016;27 (4):732-738. doi:10.1093/annonc/mdw016

17. Su S, Liu L, Li C, Zhang J, Li S. Prognostic role of pretreatment derived neutrophil to lymphocyte ratio in urological cancers: a systematic review and meta-analysis. Int $J$ Surg. 2019;72:146-153. doi:10.1016/j.ijsu.2019.10.043

18. Dalpiaz O, Luef T, Seles M, et al. Critical evaluation of the potential prognostic value of the pretreatment-derived neutrophil-lymphocyte ratio under consideration of C-reactive protein levels in clear cell renal cell carcinoma. Br J Cancer. 2017;116(1):85-90. doi:10.1038/bjc.2016.393

19. Hsieh AH, Tahkar H, Koczwara B, et al. Pre-treatment serum lactate dehydrogenase as a biomarker in small cell lung cancer. Asia Pac J Clin Oncol. 2018;14:e64-e70. doi:10.1111/ajco.12674

20. Hua Y, Liang C, Zhu J, et al. Expression of lactate dehydrogenase $\mathrm{C}$ correlates with poor prognosis in renal cell carcinoma. Tumour Biol. 2017;39(3):1010428317695968. doi:10.1177/1010428317695968

21. Dharmapuri S, Özbek U, Lin JY, et al. Predictive value of neutrophil to lymphocyte ratio and platelet to lymphocyte ratio in advanced hepatocellular carcinoma patients treated with anti-PD-1 therapy. Cancer Med. 2020;9(14):4962-4970. doi:10.1002/cam4.3135

22. Finn RS, Qin S, Ikeda M, et al. Atezolizumab plus bevacizumab in unresectable hepatocellular carcinoma. $N$ Engl J Med. 2020;382 (20):1894-1905. doi:10.1056/NEJMoa1915745

23. Finn RS, Ikeda M, Zhu AX, et al. Phase Ib study of lenvatinib plus pembrolizumab in patients with unresectable hepatocellular carcinoma. J Clin Oncol. 2020;38(26):2960-2970. doi:10.1200/ JCO. 20.00808

24. Zhu AX, Finn RS, Edeline J, et al. Pembrolizumab in patients with advanced hepatocellular carcinoma previously treated with sorafenib (KEYNOTE-224): a non-randomised, open-label Phase 2 trial. Lancet Oncol. 2018;19(7):940-952. doi:10.1016/S1470-2045(18) 30351-6

25. Greten TF, Mauda-Havakuk M, Heinrich B, Korangy F, Wood BJ. Combined locoregional-immunotherapy for liver cancer. $J$ Hepatol. 2019;70(5):999-1007. doi:10.1016/j.jhep.2019.01.027

26. Xu W, Liu K, Chen M, et al. Immunotherapy for hepatocellular carcinoma: recent advances and future perspectives. Ther Adv Med Oncol. 2019;11:1758835919862692. doi:10.1177/17588359198 2692 


\section{Publish your work in this journal}

The Journal of Hepatocellular Carcinoma is an international, peerreviewed, open access journal that offers a platform for the dissemination and study of clinical, translational and basic research findings in this rapidly developing field. Development in areas including, but not limited to, epidemiology, vaccination, hepatitis therapy, pathology

Submit your manuscript here: https://www.dovepress.com/journal-of-hepatocellular-carcinoma-journal and molecular tumor classification and prognostication are all considered for publication. The manuscript management system is completely online and includes a very quick and fair peer-review system, which is all easy to use. Visit http://www.dovepress.com/ testimonials.php to read real quotes from published authors. 